SCIENTIFIC REPORT

\title{
Interocular amplitude differences of the full field electroretinogram in normal subjects
}

Y Rotenstreich, G A Fishman, R J Anderson, D G Birch

Br J Ophthalmol 2003;87:1268-1271

Aims: To determine the interocular amplitude response difference of the electroretinogram (ERG) in normal subjects. Methods: 79 subjects, without retinal changes of clinical significance, underwent ERG testing. They included 63 men and 16 women, with a mean age of 44 (SD 12) years and range of 18-65 years. Isolated rod, scotopic maximal, dark adapted $30 \mathrm{~Hz}$ flicker, photopic single flash, and light adapted $30 \mathrm{~Hz}$ flicker responses were recorded in both eyes following the International Society for Clinical Electrophysiology of Vision (ISCEV) standard protocol. The interocular percentage differences of the ERG b-wave amplitudes were calculated and presented as percentiles (25th, 50th, 75th, 95th), means (SD), and medians.

Results: The median interocular percentage differences in the b-wave amplitudes for the above ERG stimulus responses were $10 \%, 8 \%, 10 \%, 11 \%$, and $10 \%$, respectively. The mean interocular percentage differences were $11 \%, 11 \%, 12 \%$, $13 \%$, and $14 \%$. The 95 th percentiles for the interocular percentage differences were $28 \%, 27 \%, 36 \%, 33 \%$, and $35 \%$, respectively.

Conclusions: The interocular percentage differences in the ERG b-wave amplitudes for five different stimulus responses were similar in our cohort of individuals without clinically significant retinal changes and ranged from a median of 8$11 \%$ and a 95 th percentile of $27-36 \%$. Our findings should be useful for determining sample sizes in future therapeutic trials on retinal diseases with monocular therapeutic strategies and may also have application for the more accurate detection of asymmetric retinal disease.

$\mathrm{T}$ he full field electroretinogram (ERG) is a well established procedure for investigating retinal disease. Although a standard testing protocol has been recommended by the International Society for Clinical Electrophysiology of Vision (ISCEV), ${ }^{1}$ there are limited data on the interocular difference that might be anticipated to occur with the use of the ISCEV testing protocol. ${ }^{2-5}$

In this study, we calculated the interocular difference of the b-wave amplitude for various ERG stimuli in subjects primarily without or, in isolated instances, with only localised retinal changes. Measuring the range of normal interocular difference provides an important parameter for the clinical evaluation of patients with asymmetric retinal eye diseases, such as acute zonal occult outer retinopathy, ${ }^{4}$ retinal toxicity from an intraocular metallic foreign body, ${ }^{67}$ and carriers of $\mathrm{X}$ linked retinitis pigmentosa. ${ }^{59}$ Furthermore, knowledge of interocular ERG amplitude variability is important for determining sample size in treatment trials and for monitoring possible therapeutic effects in future clinical treatment trials for hereditary retinal diseases that utilise a monocular treatment protocol.

\section{METHODS}

ERG data were collected prospectively from 79 subjects, 63 females and 16 males, who were participants in a pharmaceutical study. The subjects included those being treated with either a proton pump inhibitor or a non-proton pump inhibitor for, predominantly, gastro-oesophageal reflux disease. They also included a group of normal control subjects. The medications used had no untoward effect on ERG amplitudes when compared to the control subjects. The study was carried out with approval from the institutional review board at the University of Texas, UT Southwestern Medical Center. Seventy two subjects were white, five black, and two Asian. The mean age was 44 (SD 12) years (range 18-65 years). Each subject underwent a full ophthalmological examination, visual field evaluation (Humphrey, 30-2 with standard full threshold technique), and ERG testing in both eyes. All participants had a normal visual field, normal ERG amplitude results, and none showed an asymmetric refractive error or strabismus. Of the 79 subjects, six had peripheral lattice degeneration, one showed mild bilaterally symmetric lens opacities, one was diagnosed with presumed bilateral ocular histoplasmosis, and one with limited, bilaterally symmetric macular drusen. None of the subjects had either a systemic disease or were on any medications known to affect ERG function.

Pupils were dilated to at least a $7 \mathrm{~mm}$ diameter with $2.5 \%$ phenylephrine hydrochloride and $1 \%$ cyclopentolate hydrochloride. ERG amplitudes on both eyes of each subject were obtained. Dark adapted ERG responses after a minimum of 45 minutes for dark adaptation included an isolated rod, maximal response, and $30 \mathrm{~Hz}$ flicker. Light adapted responses included a single white flash and $30 \mathrm{~Hz}$ flicker. These recording procedures adhered to a recommended international standard for clinical electrophysiological measurements.

For each of the five stimuli, the percentage difference between right and left eyes for the ERG b-wave amplitudes in

Table 1 The interocular percentage difference in b-wave amplitude for each ERG stimulus

\begin{tabular}{|c|c|c|c|c|c|}
\hline & \multicolumn{2}{|l|}{ Photopic } & \multicolumn{3}{|l|}{ Scotopic } \\
\hline & $\begin{array}{l}\text { Single } \\
\text { flash (\%) }\end{array}$ & $\begin{array}{l}30 \mathrm{~Hz} \\
\text { flicker (\%) }\end{array}$ & $\begin{array}{l}\text { Isolated } \\
\text { (blue) (\%) }\end{array}$ & $\begin{array}{l}\text { Maximal } \\
\text { white (\%) }\end{array}$ & $\begin{array}{l}30 \mathrm{~Hz} \\
\text { flicker (\%) }\end{array}$ \\
\hline Mean (SD) & $13(10)$ & $14(11)$ & $11(9)$ & $11(9)$ & $12 \pm 9$ \\
\hline $\begin{array}{l}\text { Range } \\
\text { Percentile* }\end{array}$ & $0-54$ & $0-57$ & $0-34$ & $0-33$ & $1-44$ \\
\hline 25 th & $\leqslant 6$ & $\leqslant 6$ & $\leqslant 4$ & $\leqslant 4$ & $\leqslant 6$ \\
\hline 50tht & $\leqslant 11$ & $\leqslant 10$ & $\leqslant 10$ & $\leqslant 8$ & $\leqslant 10$ \\
\hline 75 th & $\leqslant 18$ & $\leqslant 20$ & $\leqslant 17$ & $\leqslant 17$ & $\leqslant 15$ \\
\hline $95 \mathrm{th}$ & $\leqslant 33$ & $\leqslant 35$ & $\leqslant 28$ & $\leqslant 27$ & $\leqslant 36$ \\
\hline
\end{tabular}

*A percentile represents the maximal interocular percentage difference in the percentile of the population.

tThe 50th percentile represents the median. 
Table 2 The number of individuals (\%) at different levels of percentage difference

\begin{tabular}{|c|c|c|c|c|c|}
\hline & \multicolumn{2}{|l|}{ Photopic } & \multicolumn{3}{|l|}{ Scotopic } \\
\hline & Single flash & $30 \mathrm{~Hz}$ flicker & Isolated (blue) & Maximal white & $30 \mathrm{~Hz}$ flicker \\
\hline $10 \%$ difference or lower & $35(44)$ & $40(51)$ & $44(56)$ & $46(58)$ & $41(52)$ \\
\hline $20 \%$ difference or lower & $67(85)$ & $61(77)$ & $66(84)$ & $66(84)$ & $66(84)$ \\
\hline $30 \%$ difference or lower & $74(94)$ & 69 (87) & $76(96)$ & 77 (97) & $73(92)$ \\
\hline
\end{tabular}

each subject was calculated. This difference was computed by dividing the ERG b-wave amplitude difference between the eyes by the higher amplitude. The effect of the b-wave amplitude on the interocular percentage difference was evaluated by analysing the relation between the average amplitude of both eyes to the interocular percentage difference in each subject.

\section{RESULTS}

The median percentage interocular differences of the b-wave amplitudes were similar for the photopic single flash, light adapted $30 \mathrm{~Hz}$ flicker, isolated rod, maximal scotopic, and dark adapted $30 \mathrm{~Hz}$ flicker responses $(11,10,10,8$, and 10 respectively). The maximal interocular percentage differences observed were $54,57,34,33$, and 44 , respectively. The 95 th percentiles for the percentage difference were $31,34,28,27$, and 34, respectively (Table 1 ). The number of individuals and percentage with ERG b-wave interocular amplitude differences at $\leqslant 10 \%$, $\leqslant 20 \%$, or $\leqslant 30 \%$ as well as the median differences and 95th percentile interocular ERG b-wave amplitude differences for the five stimulus conditions are shown in Tables 1 and 2 . Of all the participants, only one subject had a $21 \%$ difference or greater for each one of the stimuli.

The distribution and the cumulative curves of the interocular differences for the b-wave amplitudes obtained with the five different stimuli are presented in Figures 1-5. The cumulative curves represent the percentage of the study
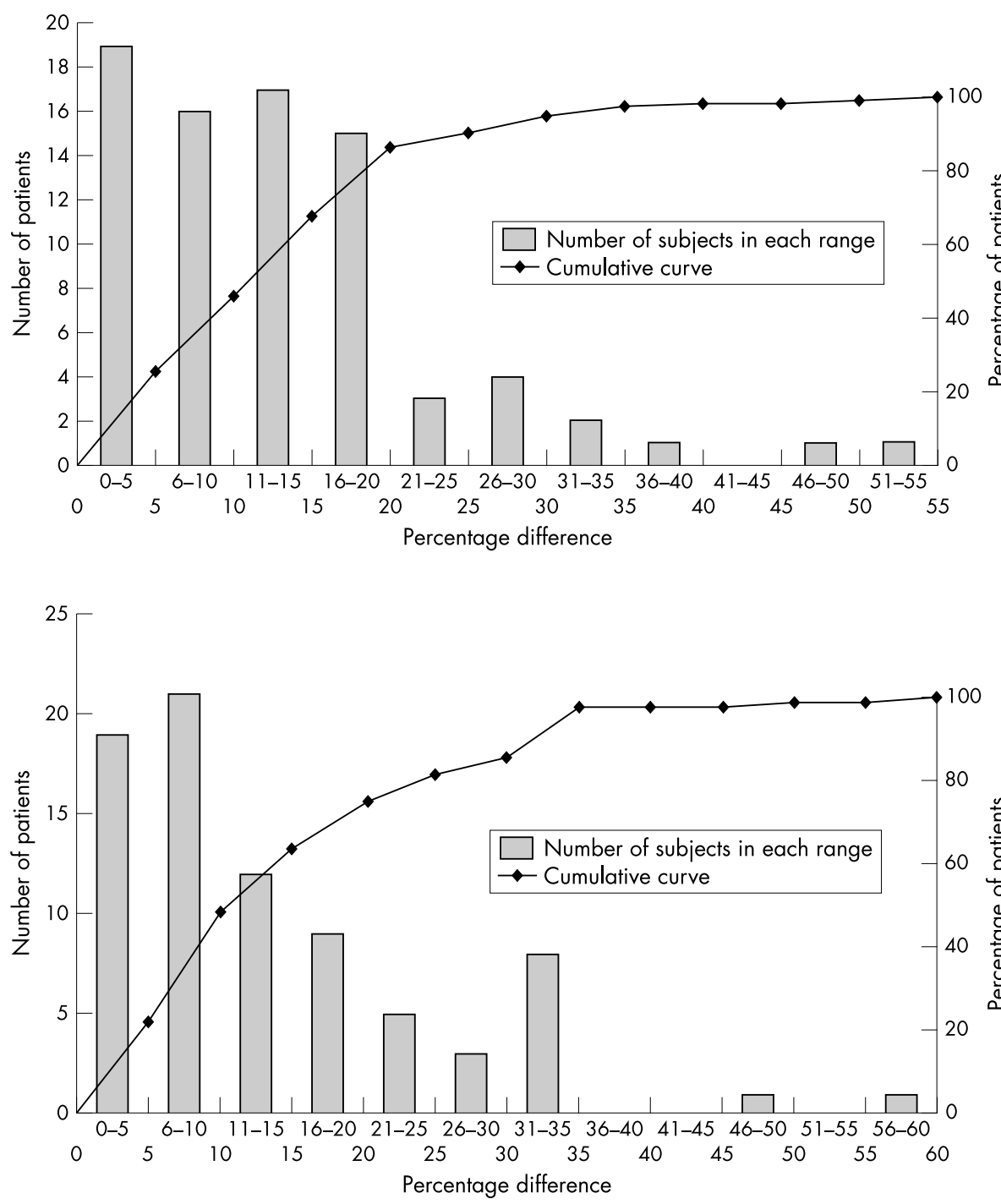

Figure 1 The columns represent the interocular percentage difference distribution in $5 \%$ intervals of the ERG b-wave amplitude responses for the single flash photopic stimulus. The line demonstrates the cumulative curve for this stimulus in $5 \%$ difference increment steps.

Figure 2 The columns represent the interocular percentage difference distribution in $5 \%$ intervals of the ERG b-wave amplitude responses for the light adapted $30 \mathrm{~Hz}$ flicker stimulus. The line demonstrates the cumulative curve for this stimulus in 5\% difference increment steps. 


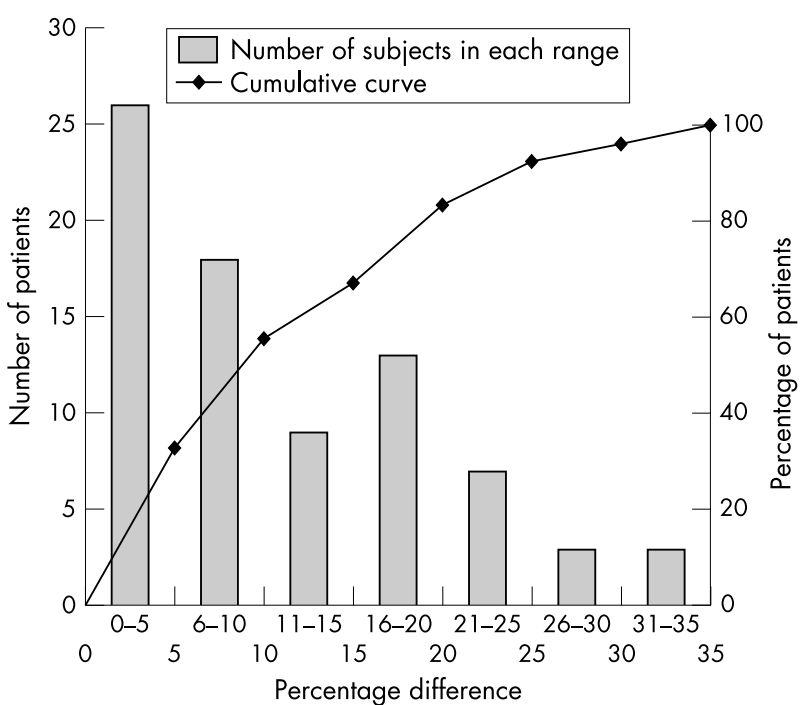

Figure 3 The columns represent the interocular percentage difference distribution in $5 \%$ intervals of the ERG b-wave amplitude responses for the scotopic isolated rod stimulus. The line demonstrates the cumulative curve for this stimulus in 5\% difference increment steps.

population at various levels of interocular percentage difference.

\section{DISCUSSION}

The determination of interocular difference in ERG b-wave amplitude responses in visually normal subjects has provided useful information for comparison with the retinal function of patients with different asymmetric retinal diseases. ${ }^{4-9}$ The aim of this study was to establish the degree of the interocular percentage difference in subjects with, at most, mild retinal changes using the ISCEV ${ }^{1}$ recommendations for various stimulus conditions. In our study, interocular percentage differences are presented as percentiles (25th, 50th, 75th, 95th), medians, and means (SD). Among our 79 subjects, the median interocular percentage differences of the ERG b-wave amplitude responses in the five different stimuli were similar. Fifty per cent of our cohort subjects had an interocular percentage difference of from $8 \%$ to $11 \%$ for different stimuli, which is consistent with an interocular amplitude difference of within $10 \%$, previously cited by

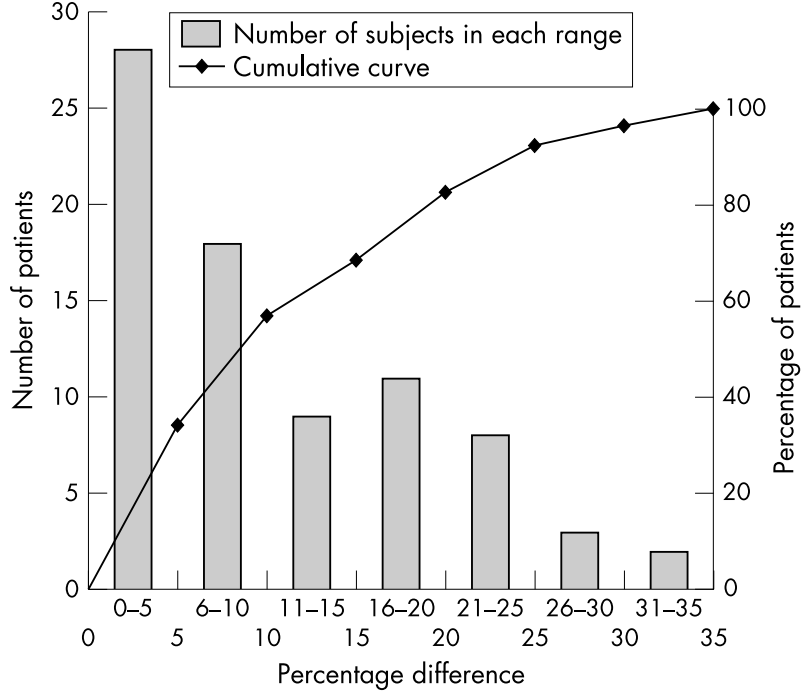

Figure 4 The columns represent the interocular percentage difference distribution in $5 \%$ intervals of the ERG b-wave amplitude responses for the scotopic maximal stimulus. The line demonstrates the cumulative curve for this stimulus in $5 \%$ difference increment steps.

Fishman et al. ${ }^{3}$ Ninety five per cent of our cohort had interocular percentage differences in the five stimuli that ranged from $27-36 \%$ or less. The average interocular percentage difference ranged from $11-14 \%$ for the five different stimuli (Table 1).

For comparison, we calculated the interocular percentage differences in ERG b-wave amplitude from the 22 normal individuals in studies by Jacobson et al. ${ }^{45}$ (Table 3 ). We found that the interocular percentage differences in the same normal population reported in these studies were, overall, comparatively smaller than in our subjects (compare Tables 1 and 3). Fifty per cent of their cohort had interocular percentage differences that ranged from $4-8 \%$ or less while $95 \%$ had a range of $12-16 \%$ or less for different stimuli. The mean interocular percentage difference ranged from $5 \%$ to $8 \%$.

In our study, we also chose arbitrary levels of percentage interocular b-wave amplitude differences $(10 \%, 20 \%$, and $30 \%$ ) to compare among different stimuli (Table 2). For the different stimulus responses, generally over $90 \%$ of our

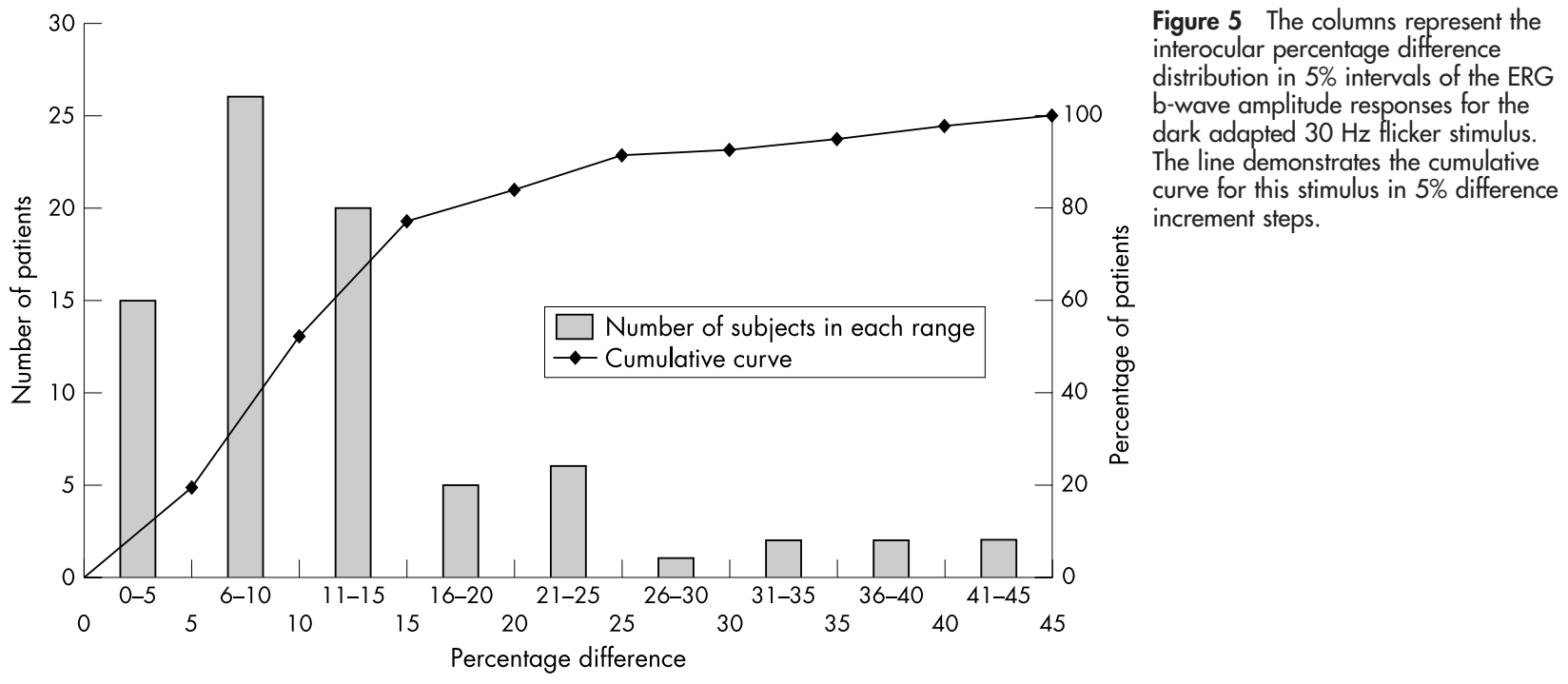

Figure 5 The columns represent the interocular percentage difference distribution in $5 \%$ intervals of the ERG for the curve for this stimulus in 5\% difference 
Table 3 Data from Jacobson et $a^{45}$ analysed for interocular percentage difference

\begin{tabular}{|c|c|c|c|c|}
\hline & \multicolumn{2}{|l|}{ Photopic } & \multicolumn{2}{|l|}{ Scotopic } \\
\hline & $\begin{array}{l}\text { Single flash } \\
(\%)\end{array}$ & $\begin{array}{l}30 \mathrm{~Hz} \text { flicker } \\
(\%)\end{array}$ & $\begin{array}{l}\text { Isolated (blue) } \\
(\%)\end{array}$ & $\begin{array}{l}\text { Maximal } \\
\text { white (\%) }\end{array}$ \\
\hline Mean (SD) & $7(6)$ & $7(4)$ & $5(4)$ & $8(9)$ \\
\hline Range & $1-25$ & $1-15$ & $1-15$ & $1-33$ \\
\hline \multicolumn{5}{|l|}{ Percentile* } \\
\hline 25 th & $\leqslant 3$ & $\leqslant 4$ & $\leqslant 2$ & $\leqslant 3$ \\
\hline 50tht & $\leqslant 5$ & $\leqslant 8$ & $\leqslant 4$ & $\leqslant 6$ \\
\hline 75 th & $\leqslant 8$ & $\leqslant 10$ & $\leqslant 7$ & $\leqslant 9$ \\
\hline 95th & $\leqslant 16$ & $\leqslant 13$ & $\leqslant 12$ & $\leqslant 16$ \\
\hline
\end{tabular}

*A percentile represents the maximal interocular percentage difference in the percentile of the population.

†The 50th percentile represents the median.

population had a $30 \%$ or less interocular difference. Our findings are consistent with a previous citation indicating that a normal interocular b-wave amplitude difference should be no greater than $20-24 \%{ }^{3}$ Figures $1-5$ show the quantification of our population at various continuous levels of interocular percentage differences.

In a study of 95 healthy individuals, Ayres et al showed that the difference between the two eyes of the same subject was smaller than the difference between the eyes of different subjects. They demonstrated that the maximal interocular difference in the ERG b-wave amplitude for the maximal scotopic response was $144.58 \mu \mathrm{V}$, which was $29 \%$ of their maximal intersubject b-wave amplitude range. For comparison, in our study these values were $163.38 \mu \mathrm{V}$ and $31 \%$, respectively.

Various technical factors, such as contact lens placement, the structural integrity of the corneal surface, pupil size, among others, may impact on the interocular difference in ERG amplitudes. Regardless of the explanation, our findings can be applied in the design of future therapeutic trials, which implement monocular therapeutic strategies for various retinal diseases, and define more accurate criteria for the detection of asymmetric retinal disease.

\section{Authors' affiliations}

Y Rotenstreich, G A Fishman, Department of Ophthalmology and Visual

Science, University of Illinois at Chicago, Chicago, IL, USA

R J Anderson, Division of Epidemiology and Biostatistics, University of Illinois at Chicago, Chicago, IL, USA

D G Birch, Retina Foundation of the Southwest, Dallas, TX, USA

Correspondence to: Professor Gerald A Fishman, 1855 W Taylor Chicago, IL 60612,USA; gerafish@uic.edu

Accepted for publication 21 January 2003

\section{REFERENCES}

1 Marmor MF, Zrenner E. Standard for clinical electroretinography (1999 update). International Society for Clinical Electrophysiology of Vision. Doc Ophthalmol 1998-99;97:143-56.

2 Ayres LM, Caruso RC, Lopez $\mathrm{P}$, et al. Diagnostic significance of interocular differences in ganzfeld electroretinogram (ERG) amplitude. Invest Ophthalmol Vis Sci 1998;39:B235, S972.

3 Fishman GA, Birch DG, Holder GE, et al. Electrophysiologic testing in disorders of the retina, optic nerve, and visual pathway. 2nd ed. San Francisco: The Foundation of the American Academy of Ophthalmology, 2001:10-11.

4 Jacobson SG, Morales DS, Sun XK, et al. Pattern of retinal dysfunction in acute zonal occult outer retinopathy. Ophthalmology 1995;102:1187-98.

5 Jacobson SG, Yagasaki K, Feuer WJ, et al. Interocular asymmetry of visual function in heterozygotes of $\mathrm{X}$-linked retinitis pigmentosa. Exp Eye Res 1989:48:679-91.

6 Schechner R, Miller B, Merksamer E, et al. Long term follow up of ocular siderosis: quantitative assessment of the electroretinogram. Doc Ophthalmol 1991;76:231-40

7 Fishman GA, Birch DG, Holder GE, et al. Electrophysiologic testing in disorders of the retina, optic nerve, and visual pathway. 2nd ed. San Francisco: The Foundation of the American Academy of Ophthalmology, 2001:115-16.

8 Fishman GA, Weinberg AB, McMahon $\Pi$. X-linked recessive retinitis pigmentosa. Clinical characteristics of carriers. Arch Ophthalmol 1986;104:1329-35.

9 Berson EL, Rosen JB, Simonoff EA. Electroretinographic testing as an aid in detection of carriers of $\mathrm{X}$-chromosome-linked retinitis pigmentosa. Am J Ophthalmol 1979;87:460-8. 